\title{
A IMPORTÂNCIA DA EDUCAÇÃO NA SAÚDE
}

\author{
ASSIS, Flávia Cristina Nunes - UFG \\ LIMA, Cláudia Regina Costa - UFG \\ SOUZA, Jéssica Yonara - UFG \\ BENITE-RIBEIRO, Sandra Aparecida - UFG
}

Resumo: Desde os tempos remotos acredita-se que o pensamento educacional seja de grande importância nos diversos setores da sociedade, sendo alvo de constante reflexão de estudiosos da área. O pensamento educacional na saúde foi proposto tardiamente embora a populaçao carecesse de idéias e açoes que visassem educar para previnir, buscando uma melhora na qualidade de vida da população, pois muitas doenças eram adquiridas por falta de informação. No século XX a população era alvo de epidemias como por exemplo febre amarela e varíola o que despertou a preocupaçao dos profissionais de saúde e da classe governamental, fazendo com que nomes como Oswaldo Cruz tivessem sua importância neste contexto. Propostas educacionais em que profissionais de saúde ouvem e educam pequenos grupos de pessoas são uma opçao para o desenvolvimento dessas políticas educacionais, supostamente esses profissionais são os mais indicados para tais atividades educacionais pois são os maiores portadores dos conhecimentos práticos e da realidade da saúde, podendo esclarecer e orientar da melhor maneira a população que carece desse sistema educacional, porém uma dificuldade vêm sendo encontrada para essa prática pois esses profissionais apresentam uma carência na didática.

Palavras chaves: Educação, saúde.

\footnotetext{
Abstract: Since ancient times believed that the educational thought has a great importance in various sectors of society, the target of constant reflection of scholars in the area. The educational thought in health was proposed late although in need of ideas and actions that serve to educate to prevent, seeking a better quality of population's life, because many diseases are acquired by lack of information. In the twentieth century the population was subject to epidemics such as yellow fever and smallpox which raised the
} 
concern of health professionals and government class, making names like Oswaldo Cruz had their importance in this context. Educational proposals in wich health professionals listen and educate small groups of people are one option for the development of educational policies, supposedly these professioals are the most suitable for such esucational activities because they are the largest holders of practical knowledge and of the reality of health, they may clarify and guide people who need educational system to the best manner to conduct, but a difficulty have been found to this practice because these professionals have a lack in teaching.

Keywords: Education, health.

\section{Considerações iniciais}

O pensamento educacional existe desde os tempos remotos, sendo que vários pensadores como João Pestalozzi OJOJ e Lev S. Vygotsky (1896-1934) sugeriram várias teorias, as quais, apesar de sujeitas às críticas, contribuem no pensamento educacional dos dias atuais. (CARVALHO, 2009).

Pestallozzi (TRINDADE, 2008) exerceu grande influência no pensamento educacional e foi adepto da educação pública. Ele promoveu um processo de democratização da educação, pois defedia que toda criança tem o direito de "desenvolver os poderes dado por Deus". Assim, influenciou governantes a se interessarem pela educação das crianças das classes menos favorecidas. Ele argumentava que a escola deveria assemelhar-se a uma casa bem organizada, pois afirmava que o lar é a maior instituição de educação, sendo base para a formação moral, política e religiosa. Segundo Pestallozzi, somente a educação poderia mudar a terrível condição de vida do povo, visão esta aceita atualmente, até mesmo por muitos governantes (TRINDADE, 2008).

Posteriormente, Vygotsky (OLIVEIRA, 1997) construiu sua teoria tendo por base o desenvolvimento do indivíduo como resultado de um processo sócio-histórico. Sua questão central é a aquisição de conhecimentos pela interação do sujeito com o meio em que vive, pois acreditava que o sujeito não é apenas ativo, mas interativo, porque constrói conhecimentos e se constitui a partir de relações intra e interpessoais. Vygotsky 
advogava que as relações entre pensamento, linguagem e a questão cultural, teriam grande influência no processo de construção de significados pelos indivíduos, e a escola teria o papel na transmissão de conhecimento, que é de natureza diferente daqueles aprendidos na vida cotidiana. Afirmava que a escola é o lugar onde a intervenção pedagógica desencadeia o processo ensino-aprendizagem, (OLIVEIRA, 1997).

Mais recentemente, Paulo Freire (1921-1997) elaborou uma concepção problematizadora da educação, na qual afirma que o conhecimento não pode advir de um ato de "doação" que o educador faz ao educando, mas sim, um processo que se realiza pelo contato do homem com o mundo vivenciado, o qual não é estático, mas dinâmico e em transformação contínua.

"...e educador já não é aquele que apenas educa, mas o que, enquanto educa, é educado, em diálogo com o educando, que ao ser educado, também educa" (GADOTTI, 2000).

Tendo grandes influências nos pensamentos educacionais atuais, essas e muitas outras teorias ajudaram a compor a definição de educação que temos hoje. Segundo o Novo Dicionário da Língua Portuguesa, de Aurélio Buarque de Holanda Ferreira (1988), educação é o

"Processo de desenvolvimento da capacidade física, intelectual ou moral da criança e do ser humano em geral, visando à sua melhor integração individual e social".

De forma mais específica, a educação em saúde consiste basicamente no planejamento e na execução de atividades que visam prevenir doenças e suprimir carências das populações, como práticas inadequadas de higiene, que ignoram a importância de cuidados básicos para prevenção e controle de epidemias (CANDEIAS, 1997).

Assim, a prática da educação em saúde preconiza desenvolver nas pessoas juízo crítico e capacidade de intervenção sobre suas vidas e sobre o ambiente com o qual interagem (LEVY 1997). As informações e orientações devem ser expressas de maneira clara e sem restrições, de forma a garantir boa compreensão pelos sujeitos para que os objetivos e metas sejam alcançados (L'ABBATE, 1994). 
Como a educação em saúde depende de um conjunto de saberes e práticas inerentes aos profissionais da saúde e de algumas áreas da educação, são esses os agentes responsáveis pela orientação da população para adoção de hábitos considerados saudáveis, tais como higiene pessoal e ambiental, alimentação adequada, abandono do sedentarismo, do tabagismo etc (ALVES, 2005).

As doenças cardiovasculares e crônicas como hipertensão, diabetes e obesidade são um importante problema de saúde pública e a principal causa de morte da população adulta dos países desenvolvidos (TRINDADE, 1998). A educação em saúde é, portanto, imprescindível, pois não é possível o controle adequado dessas doenças se o paciente não for instruído a seguir os princípios em que se fundamentam seu tratamento. A participação ativa do indivíduo é a única solução eficaz no controle dessas doenças e na prevenção de suas complicações (PAIVA, 2006).

Assim, no presente trabalho pretende-se discutir os aspectos relacionados à implantação das políticas de educação para a promoção de saúde, as ações desenvolvidas e os papéis dos profissionais da saúde na orientação da população sobre hábitos de vida considerados saudáveis, que incluem alimentação equilibrada e exercício físico regular, além de tratamento terapêutico correto de hipertensos e diabéticos.

\section{Histórico das políticas de saúde}

As políticas de educação para a saúde e as ações preventivas surgiram tardiamente nas sociedades contemporâneas e são ainda mais recentes nos países em desenvolvimento. Atualmente, a atenção à educação para a saúde está relativamente bem estabelecida em países desenvolvidos. Entretanto, em países como o Brasil, principalmente em estados menos desenvolvidos econômica e socialmente, as ações preventivas ainda não estão bem instauradas (ALVES, 2005).

Na Europa, durante a segunda metade do século XIX, com o processo de industrialização, houve uma explosão populacional na zona urbana, principalmente das classes menos favorecidas. As pessoas geralmente se agrupavam e viviam em cortiços, nos quais as condições de higiene e saneamento básico eram precários, propiciando o surgimento de epidemias como peste negra, cólera, gripe espanhola, lepra e outras que, em seguida, atingiriam as classes dominantes. Assim, houve a implantação de ações 
disciplinantes das classes populares com difusão de regras de higiene e de condutas morais. Exercícios de controle social e sanitário foram utilizados na Europa do século XIX como ações para controle dessas epidemias (COSTA, 1987).

No Brasil, as ações voltadas para a disciplinarização higiênica só ocorreram no início do século XX, tendo como focos de atuação os hospitais, os hospícios, as prisões e as escolas. As epidemias de varíola, peste, febre amarela, tuberculose, entre outras, foram importantes estímulos para o desenvolvimento de políticas de educação e saúde, principalmente dentre as classes menos favorecidas. Nesse período, destacou-se a política sanitária liderada por Oswaldo Cruz, que implantou a vacinação compulsória e a vigilância do comportamento das classes menos favorecidas, no que diz respeito ao saneamento básico e higiênico, visando o controle das epidemias (ALVES, 2005).

Essa política de educação partia do pressuposto de que a conscientização de indivíduos para boas práticas higiênicas e sanitárias, como o simples ato de lavar as mãos, contribuiriam para melhoria da qualidade de vida e controlaria doenças infecto contagiosas, muitas vezes consideradas graves (ALVES, 2005).

A partir do final da década de 40 , foram desenvolvidas práticas educativas mais voltadas para comunidade e menos individualistas, buscando maior eficácia e disseminação da política preventiva (VASCONCELOS, 2001), como as críticas à centralização das ações de saúde e a defesa da ampliação dos municípios, além da necessidade de melhor aparelhar os serviços sanitários (OLIVEIRA, 2009).

Durante o regime militar na década de 60 a educação em saúde correspondeu ao controle sobre as pessoas (SMEKE \& OLIVEIRA - 2001), o que despertou insatisfação e resistência da população. Isso levou ao surgimento de movimentos sociais na década de 70 que reuniram intelectuais e cidadãos. Tinham como objetivo a ruptura do autoritarismo nas práticas educativas, destacando-se o movimento Educação Popular em Saúde (MOPS), o qual incentivava a iniciativa de profissionais da saúde nos serviços que prestavam assistência às classes populares. Esse movimento integrava-se a projetos mais amplos, dentre os quais predominava a metodologia da Educação Popular (VASCONCELOS, 2001). Desde então, os pacientes passaram a ter um acompanhamento mais amplo no processo saúde-doença-cuidado.

Baseados nesse movimento de Educação Popular em Saúde, países como o Brasil, que até a década de 70 adotavam como política de saúde imposições de regras, 
passaram a aderir a novos modelos de saúde pública. Surgem então projetos e instituições populares com técnicos na área de saúde, inicialmente sem vínculos políticos movidos por ações voluntárias. Com a abertura política, esses projetos obtiveram o apoio popular e se expandiram mais facilmente (LIMA, 2006). A experiência ocorrida na zona leste da cidade de São Paulo é o exemplo mais conhecido, mas o Movimento Popular de Saúde chegou influenciar todo o território nacional (VASCONCELOS, 2001).

Se no passado, parasitoses e doenças relacionadas à falta de higiene constituíam a principal causa de morte da população mundial e brasileira determinando as principais ações de saúdes a serem realizadas, no presente são as doenças relacionadas à obesidade que começam a dominar o cenário das principais patologias que resultam em óbitos. Assim, a educação para o desenvolvimento de melhores hábitos de vida tem fundamental importância para prevenção e tratamento dessas doenças (ALVES e NUNES, 2006).

\section{Educação para prevenção de doenças}

Tem sido demonstrado que a existência de maus hábitos alimentares e do sedentarismo podem elevar a probabilidade do desenvolvimento de sobrepeso e/ou obesidade (BERTHOUD et al., 2008; BJORNTORP, 1997; BLUNDELL et al., 1999; BUTTE et al., 2003; CUEVAS et al., 2009) que, por sua vez, são fatores de risco de doenças crônicas, tais como hipertensão arterial e doenças cardiovasculares (CHO et al., 2002; COLLE et al., 2008; HALL et al., 2000; MANSON et al., 1990; MISRA et al., 2003; RIBEIRO et al., 2003), síndrome metabólica (CUEVAS et al., 2009; DALLMAN et al., 2007; GUIMARAES et al., 2008; HU et al., 2006; OGDEN et al., 2007; ROSMOND, 2005) e diabetes (ADEGHATE et al., 2006; BRINDLEY et al., 1989; HU et al., 2004; LAAKSONEN et al., 2002; MORAN et al., 2003; SCHWARTZ et al., 2008).

A hipertensão arterial (HA) que consiste, de forma geral, na elevação anormal da pressão nas artérias acima de 140x90 mmHg (milímetros de mercúrio) em adultos, é uma doença multifatorial (CHOBANIAN et al., 2003), representando importante fator de risco para a ocorrência de doenças cardiovasculares (WANG et al., 2006; 
WHITWORTH, 2005), assim como de outros sistemas (BLUMENTHAL et al., 1993; QUACK et al., 2009). Além disso, é uma doença altamente incidente na sociedade moderna, acometendo cerca de 15 a $20 \%$ da população adulta e cerca de $50 \%$ dos idosos. A prevenção e o tratamento continuado são de extrema importância para o sucesso do controle da pressão arterial e das doenças relacionadas (OMS 2008).

Dentre as doenças metabólicas causadas pelos maus hábitos, pode-se destacar o Diabetes mellitus tipo II, que é caracterizado por resistência à insulina nos tecidos periféricos resultando em hiperglicemia e demais distúrbios metabólicos. Estima-se que 60 a $90 \%$ dos portadores da doença apresenta obesidade ou sobrepeso, sendo o aumento do tecido adiposo o principal fator de risco para o desenvolvimento da patologia. (Sociedade Brasileira de Diabetes - S.D.B., 2009) (BRINDLEY et al., 1989; CUEVAS et al., 2009; MORAN et al., 2003; OGDEN et al., 2007).

De acordo com o relatório da Organização Mundial de Saúde (OMS, 2003), o cuidado às condições crônicas, como hipertensão e diabetes, tem constituído um importante desafio aos sistemas de saúde (Alves e Nunes, 2006). Segundo a OMS (2008), 80\% das mortes prematuras causadas por essas doenças podem ser evitadas por meio de mudanças no estilo de vida, como exemplo a prática de atividade física regular (BLUMENTHAL et al., 2000; DENGEL et al., 1998; GEORGIADES et al., 2000; MAYER et al., 1967), dieta equilibrada (DICKINSON et al., 2009; FOGELHOLM et al., 1999; HU et al., 2006; ROSS et al., 2000) e abandono do tabagismo; medidas que juntamente com controle da ingestão de bebidas alcoólicas, são recomendadas pela Countrywide Noncommunicable Disease Intervention (CINDI) para o controle dos fatores de risco biológico associados à hipertensão, obesidade e anormalidades no metabolismo dos carboidratos e dos lipídeos (OMS, 2004).

Considerando-se a importância dos hábitos saudáveis de vida, o maior desafio para os profissionais de saúde é manter a adesão de indivíduos com doenças metabólicas e cardiovasculares crônicas, que oferecem resistência as mudanças de hábitos e de alimentação. Muitas vezes essa resistência advém da falta de conhecimento da doença e sua forma de tratamento assim como fatores sociais e econômicos (Peres et.al, 2003).

Para a manutenção do tratamento correto e ininterrupto são necessários grupos assistenciais de orientação à pacientes hipertensos e diabéticos. Os grupos devem 
almejar o esclarecimento e a adesão dos pacientes para os cuidados necessários ao controle das doenças e prevenção de enfermidades decorrentes da hipertensão e do diabetes. Deve esclarecer sobre a importância do tratamento médico, na utilização correta e controlada de fármacos e, talvez o mais importante, nas orientações para uma alimentação com maior qualidade nutricional, além da prática regular de exercícios físicos (Paiva, 2006).

\section{Dificuldades para implantação de grupos educativos}

Os principais problemas de saúde identificados quanto ao modelo assistencial durante VIII Conferência Nacional de Saúde foram:

"desigualdade no acesso ao sistema de saúde, inadequação dos serviços às necessidades, qualidade insatisfatória dos serviços e ausência de integralidade das ações" (Paim, 2003 p. 567).

No Brasil, a saúde é um direito social, inscrito na Constituição Federal de 1988, que também instituiu o Sistema Único de Saúde, como meio de concretizar esse direito (LUCCHESE, 2006). Mesmo com essa lei, a saúde pública não contribui adequadamente com as classes menos favorecidas, pois não oferece condições apropriadas para o desenvolvimento de políticas educacionais eficientes a essa população. A carência de centros médicos, profissionais, aparelhamento, medicamentos e muitas vezes maior atenção dos profissionais de saúde para com esses indivíduos promove déficits no controle e no tratamento das doenças.

A educação em saúde representa uma estratégia promissora para enfrentar os múltiplos problemas de saúde que afetam as populações humanas. Partindo de uma concepção ampla do processo saúde-doença e de seus determinantes (BUSS, 2000). Porém essa estratégia pode não ser suficiente para a correta orientação de pacientes, haja visto que os profissionais da área de saúde, que supostamente são os mais aptos para exercerem essa atividade, nem sempre sabem a melhor maneira de repassar seu conhecimento à população por terem carências didáticas em sua formação (BARATA, 1987).

De acordo com Trezza (2007), mesmo que para ser educador no desenvolvimento de atividades do cotidiano não seja preciso tornar-se especialista em teorias da educação, em teorias pedagógicas, ou em teoria da comunicação, é preciso 
entender a existência de relações fundamentais entre educação e sociedade, visto que grande parte do trabalho do profissional de saúde envolve transmissão de conhecimentos e orientações:

"Os profissionais/alunos devem sentir-se o tempo todo sujeitos do processo educativo e, da mesma maneira, aprender a considerar sujeitos o usuário e os outros profissionais" (Trezza, 2007).

Segundo Barata (1987), por parte das universidades e das escolas de saúde pública é necessário que haja uma valorização das atividades relacionadas ao ensino, frequentemente encaradas como secundárias em relação à pesquisa. Neste sentido, dar atenção à educação como objeto de estudo deveria ser preocupação de todo pesquisador/professor da área de saúde

\section{Conclusão}

A necessidade da adoção de novas formas educativas visando melhor orientação da população existe e demanda maior atenção das autoridades e dos profissionais da área de saúde, pois é um método útil e eficiente na prevenção e tratamento de doenças crônicas, como a hipertensão e o diabetes que apresentam alta incidência nas sociedades contemporâneas.

A prática de educação em saúde começou tardiamente no Brasil, assim, pode-se dizer que ainda estamos a caminho de um país no qual a prevenção e a educação para a saúde tenham a importância e a atenção que merecem.

Os profissionais da área de saúde atuam nos processos educativos, como proferimento de palestras ou ações de conscientização à população, porém podem encontrar dificuldades pela suas carências didáticas, apesar dessas dificuldades esse profissional supostamente ainda é o mais incado para essa atividade pois possui um maior conhecimento prático e das realidades da saúde.

\section{Referências Bibliográficas:}

CARVALHO, J.O e CARVALHO, L. R. S. O. A educação social no Brasil: contribuições para o debate.. In: I CONGRESSO INTERNACIONAL DE PEDAGOGIA SOCIAL, 1., 2006, . Proceedings online... Faculdade de Educação, Universidade de São Paulo. 
OLIVEIRA, M.K. Vygotsky - Aprendizado e Desenvolvimento - Um processo sóciohistórico. São Paulo, Scipione,1997.

TRINDADE, S.; e MENEZES, I. A educação na modernidade e a modernidade da escola no Brasil: séculos XIX e inicio do século XX. In Cultura Escolar Migrações e Cidadania Actas do VII Congresso LUSOBRASILEIRO de História da Educação 20 -23 Junho 2008, Porto: Faculdade de Psicologia e Ciências da Educação (Universidade do Porto).

GADOTTI, M. Perspectivas atuais da educação. São Paulo Perspec., São Paulo, v. 14 , n. 2, June 2000.

CANDEIAS, N.M.F.Conceitos de educação e de promoção em saúde: mudanças individuais e mudanças organizacionais Rev. Saúde Pública. São Paulo, vol. 31 no. 2 , Apr. 1997.

L'ABBATE, S. Educação em saúde: uma nova abordagem. Cad. Saúde Pública, Rio de Janeiro, v. 10, n. 4, Dec. 1994.

COSTA, N.R. Estado, educação e saúde: A higiene da vida cotidiana. Cad. Cedes, n.4, p. 5- 27, 1987.

ALVES, V. S. Um modelo de educação em saúde para o Programa Saúde da Família: pela integralidade da atenção e reorientação do modelo assistencial. Interface (Botucatu), Botucatu, v. 9, n. 16, Feb. 2005.

ALVES, V. S. e NUNES, M.O. Educação em saúde na atenção médica ao paciente com hipertensão arterial no Programa Saúde da Família, Interface (Botucatu), Botucatu, v.10, n.19 Jan./June. 2006.

VASCONCELOS, E. M. A saúde nas palavras e nos gestos: reflexões da rede de educação popular em saúde. Hucitec, São Paulo, 281p, 2001.

OLIVEIRA, M.C.M e VOLGT, A.M.C, Saberes e práticas na saúde: a profissionalização no ensino superior. Revista Iluminart do IFSP, São Paulo, Vol 1, no 2, Ago.2009.

SMEKE, E. L. M.; OLIVEIRA, N. L. S. Educação em saúde e concepções de sujeito. In: VASCONCELOS, E. M. (Org.) A saúde nas palavras e nos gestos: reflexões da rede educação popular e saúde. São Paulo: HUCITEC, 2001. p.115-36.

LIMA, J. C, História das lutas sociais por saúde no Brasil. Trabalho necessário. Vol 4, no 4, 2006.

PERES, D.S; MAGNA, J.M; VIANA, L.A. Portador de hipertensão arterial: atitudes, crenças, percepções, pensamentos e práticas. Rev. Saúde Pública, São Paulo, v. 37 , n. 5 , Oct. 2003 . 
PAIVA, D.C.P.; BERSUSA, A.A.S; ESCUDER, M. M. L. Avaliação da assistência ao paciente com diabetes e/ou hipertensão pelo Programa Saúde da Família do Município de Francisco Morato, São Paulo, Brasil. Cad. Saúde Pública, Rio de Janeiro, v. 22, n. 2, Feb. 2006 .

PAIM, J.S. Modelos de atenção e vigilância da saúde. In: ROUQUAYROL. M.Z.; ALMEIDA FILHO, N. (Orgs.) Epidemiologia e saúde. 6.ed. Rio de Janeiro: MEDSI, 2003a. p.567-86.

LUCCHESE, G. A Vigilância Sanitária no Sistema Único de Saúde. In: DE SETA, M.H.; PEPE, V. L. E.; OLIVEIRA, G.O. (Orgs.). Gestão e Vigilância Sanitária: modos atuais do pensar e fazer. Rio de Janeiro: Fiocruz, 2006. p.33-48.

BUSS, P. M. Promoção da saúde e qualidade de vida. Ciênc. saúde coletiva, Rio de Janeiro, v. 5, n. 1, 2000.

BARATA, P. Profissional em saúde, amador em educação. Cad. Saúde Pública, Rio de Janeiro, v. 3, n. 1, Mar. 1987.

TREZZA, M.C.S.F.; SANTOS, R.M.; SANTOS, J.M. Trabalhando educação popular em saúde com a arte construída no cotidiano da enfermagem: um relato de experiência. Texto contexto - enferm., Florianópolis, v. 16, n. 2, June 2007.

HOLANDA, A. B., 1988, Dicionário Aurélio Escolar da Língua Portuguesa, 1 ed., Editora Nova Fronteira, Rio de Janeiro, RJ

LEVY, S.N., et al. Educação em saúde: histórico, conceitos e propostas. Ministério da Saúde Diretoria de Programas de Educação em Saúde, Disponível em: http://www.datasus.gov.br/cns/temas/educacaosaude/educacaosaude.htm. Acesso em: 09/10/2009.

ADEGHATE, E., P. SCHATTNER e E. DUNN, An update on the etiology and epidemiology of diabetes mellitus. Ann N Y Acad Sci, v.1084, Nov, p.1-29, 2006.

BERTHOUD, H. R. e C. MORRISON, The brain, appetite, and obesity. Annu Rev Psychol, v.59, p.55-92, 2008.

BJORNTORP, P., Neuroendocrine factors in obesity. J Endocrinol, v.155, n.2, Nov, p.193-5, 1997.

BLUMENTHAL, J. A., D. J. MADDEN, T. W. PIERCE, W. C. SIEGEL e M. APPELBAUM, Hypertension affects neurobehavioral functioning. Psychosom Med, v.55, n.1, Jan-Feb, p.44-50, 1993.

BLUMENTHAL, J. A., A. SHERWOOD, E. C. GULLETTE, M. BABYAK, R. WAUGH, A. GEORGIADES, L. W. CRAIGHEAD, D. TWEEDY, M. FEINGLOS, M. APPELBAUM, J. HAYANO e A. HINDERLITER, Exercise and weight loss reduce blood pressure in men and women with mild hypertension: effects on cardiovascular, 
metabolic, and hemodynamic functioning. Arch Intern Med, v.160, n.13, Jul 10, p.1947-58, 2000.

BLUNDELL, J. E. e N. A. KING, Physical activity and regulation of food intake: current evidence. Med Sci Sports Exerc, v.31, n.11 Suppl, Nov, p.S573-83, 1999.

BRINDLEY, D. N. e Y. ROLLAND, Possible connections between stress, diabetes, obesity, hypertension and altered lipoprotein metabolism that may result in atherosclerosis. Clin Sci (Lond), v.77, n.5, Nov, p.453-61, 1989.

BUTTE, N. F. e K. J. ELLIS, Comment on "Obesity and the environment: where do we go from here?" Science, v.301, n.5633, Aug 1, p.598; author reply 598, 2003.

CHO, E., J. E. MANSON, M. J. STAMPFER, C. G. SOLOMON, G. A. COLDITZ, F. E. SPEIZER, W. C. WILLETT e F. B. HU, A prospective study of obesity and risk of coronary heart disease among diabetic women. Diabetes Care, v.25, n.7, Jul, p.1142-8, 2002.

CHOBANIAN, A. V., G. L. BAKRIS, H. R. BLACK, W. C. CUSHMAN, L. A. GREEN, J. L. IZZO, JR., D. W. JONES, B. J. MATERSON, S. OPARIL, J. T. WRIGHT, JR. e E. J. ROCCELLA, Seventh report of the Joint National Committee on Prevention, Detection, Evaluation, and Treatment of High Blood Pressure. Hypertension, v.42, n.6, Dec, p.1206-52, 2003.

COLLE, B. e S. BRUSAFERRO, [Cardiovascular risk reduction: impact of an international project]. Ann Ig, v.20, n.3 Suppl 1, May-Jun, p.43-8, 2008.

CUEVAS, A., V. ALVAREZ e C. OLIVOS, The emerging obesity problem in Latin America. Expert Rev Cardiovasc Ther, v.7, n.3, Mar, p.281-8, 2009.

DALLMAN, M. F., S. F. AKANA, N. C. PECORARO, J. P. WARNE, S. E. LA FLEUR e M. T. FOSTER, Glucocorticoids, the etiology of obesity and the metabolic syndrome. Curr Alzheimer Res, v.4, n.2, Apr, p.199-204, 2007.

DENGEL, D. R., J. M. HAGBERG, R. E. PRATlEY, E. M. ROGUS e A. P. GOLDBERG, Improvements in blood pressure, glucose metabolism, and lipoprotein lipids after aerobic exercise plus weight loss in obese, hypertensive middle-aged men. Metabolism, v.47, n.9, Sep, p.1075-82, 1998.

DICKINSON, K. M., J. B. KEOGH e P. M. CLIFTON, Effects of a low-salt diet on flow-mediated dilatation in humans. Am J Clin Nutr, v.89, n.2, Feb, p.485-90, 2009.

FOGELHOLM, M., K. KUKKONEN-HARJULA e P. OJA, Eating control and physical activity as determinants of short-term weight maintenance after a very-low-calorie diet among obese women. Int J Obes Relat Metab Disord, v.23, n.2, Feb, p.203-10, 1999.

GEORGiades, A., A. SHERWOOD, E. C. GUllette, M. A. BABYAK, A. HINDERLITER, R. WAUGH, D. TWEEDY, L. CRAIGHEAD, R. BLOOMER e J. A. 
BLUMENTHAL, Effects of exercise and weight loss on mental stress-induced cardiovascular responses in individuals with high blood pressure. Hypertension, v.36, n.2, Aug, p.171-6, 2000.

GUIMARAES, I. C., A. MOURA DE ALMEIDA e A. C. GUIMARAES, Metabolic syndrome in Brazilian adolescents: the effect of body weight. Diabetes Care, v.31, n.2, Feb, p.e4, 2008.

HALL, J. E., M. W. BRANDS, D. A. HILDEBRANDT, J. KUO e S. FITZGERALD, Role of sympathetic nervous system and neuropeptides in obesity hypertension. Braz J Med Biol Res, v.33, n.6, Jun, p.605-18, 2000.

HU, G., T. A. LAKKA, H. M. LAKKA e J. TUOMILEHTO, Lifestyle management in the metabolic syndrome. Metab Syndr Relat Disord, v.4, n.4, Winter, p.270-86, 2006.

HU, G., J. LiNDSTROM, T. T. VALLE, J. G. ERIKSSON, P. JOUSILAHTI, K. SILVENTOINEN, Q. QIAO e J. TUOMILEHTO, Physical activity, body mass index, and risk of type 2 diabetes in patients with normal or impaired glucose regulation. Arch Intern Med, v.164, n.8, Apr 26, p.892-6, 2004.

LAAKSONEN, D. E., H. M. LAKKA, L. K. NISKANEN, G. A. KAPLAN, J. T. SALONEN e T. A. LAKKA, Metabolic syndrome and development of diabetes mellitus: application and validation of recently suggested definitions of the metabolic syndrome in a prospective cohort study. Am J Epidemiol, v.156, n.11, Dec 1, p.1070-7, 2002.

MANSON, J. E., G. A. COLDITZ, M. J. STAMPFER, W. C. WILLETT, B. ROSNER, R. R. MONSON, F. E. SPEIZER e C. H. HENNEKENS, A prospective study of obesity and risk of coronary heart disease in women. N Engl J Med, v.322, n.13, Mar 29, p.882-9, 1990.

MAYER, J. e D. W. THOMAS, Regulation of food intake and obesity. Science, v.156, n.773, Apr 21, p.328-37, 1967.

MISRA, A. e N. K. VIKRAM, Clinical and pathophysiological consequences of abdominal adiposity and abdominal adipose tissue depots. Nutrition, v.19, n.5, May, p.457-66, 2003.

MORAN, O. e M. PHILLIP, Leptin: obesity, diabetes and other peripheral effects--a review. Pediatr Diabetes, v.4, n.2, Jun, p.101-9, 2003.

OGDEN, C. L., S. Z. YANOVSKI, M. D. CARROLL e K. M. FLEGAL, The epidemiology of obesity. Gastroenterology, v.132, n.6, May, p.2087-102, 2007.

QUACK, I. e L. C. RUMP, [Kidney and hypertension.]. Internist (Berl), Mar 26, 2009. 
RIBEIRO, J., S. GUERRA, A. PINTO, J. OLIVEIRA, J. DUARTE e J. MOTA, Overweight and obesity in children and adolescents: relationship with blood pressure, and physical activity. Ann Hum Biol, v.30, n.2, Mar-Apr, p.203-13, 2003.

ROSMOND, R., Role of stress in the pathogenesis of the metabolic syndrome. Psychoneuroendocrinology, v.30, n.1, Jan, p.1-10, 2005.

ROSS, R., D. DAGNONE, P. J. JONES, H. SMITH, A. PADDAGS, R. HUDSON e I. JANSSEN, Reduction in obesity and related comorbid conditions after diet-induced weight loss or exercise-induced weight loss in men. A randomized, controlled trial. Ann Intern Med, v.133, n.2, Jul 18, p.92-103, 2000.

SCHWARTZ, M. S. e A. CHADHA, Type 2 diabetes mellitus in childhood: obesity and insulin resistance. J Am Osteopath Assoc, v.108, n.9, Sep, p.518-24, 2008.

WANG, W., E. T. LEE, R. R. FABSITZ, R. DEVEREUX, L. BEST, T. K. WELTY e B. V. HOWARD, A longitudinal study of hypertension risk factors and their relation to cardiovascular disease: the Strong Heart Study. Hypertension, v.47, n.3, Mar, p.403-9, 2006.

WHITWORTH, J. A., Blood pressure and control of cardiovascular risk. Vasc Health Risk Manag, v.1, n.3, p.257-60, 2005. 